\title{
ASSESSING C2 COMMUNICATIONS FOR UAS TRAFFIC MANAGEMENT
}

\author{
Robert J. Kerczewski \\ Rafael D. Apaza \\ Alan N. Downey \\ John Wang \\ NASA Glenn Research Center, Cleveland, Ohio \\ Konstantin J. Matheou \\ Zin Technologies, Inc., Brook Park, Ohio
}

\begin{abstract}
The National Aeronautics and Space Administration's (NASA) Unmanned Aircraft Systems (UAS) Traffic Management (UTM) project works to develop tools and technologies essential for safely enabling civilian low-altitude UAS operations. Currently there is no established infrastructure to enable and safely manage the widespread use of lowaltitude airspace and UAS operations, regardless of the type of UAS. The UTM technical challenge will develop comprehensive and validated airspace operations and integration requirements to safely enable large-scale persistent access to visual line of sight and autonomous beyond visual line of sight small UAS in low-altitude airspace. Within the UTM project, a number of communications technologies to support UTM command and control (C2) are under investigation. In particular, commercial networked cellular systems are being tested and assessed for their ability to meet the reliability, scalability, cybersecurity and redundancy required. NASA Glenn Research Center is studying some of the aspects of employing such networks for UTM C2 communications. This includes the development of a test platform for sensing and characterizing the airborne $\mathrm{C} 2$ communications environment at various altitudes and in various terrains and topologies, measuring such aspects as received signal strength and interference. System performance aspects such as latency in the link, handover performance, packet error loss rate, drop outs, coverage gaps and other aspects impacting UTM operation will also be assessed. In this paper we explore some of the $\mathrm{C} 2$ approaches being proposed and demonstrated in the UTM project, the reliability, availability and other general $\mathrm{C} 2$ performance requirements, and approaches to evaluating and analyzing UTM C2 links based on commercial cellular networks.
\end{abstract}

\section{Introduction}

Small unmanned aircraft systems (sUAS), popularly referred to as "drones", have quickly proliferated in numbers and in the breadth of potential applications, such that millions are expected to be in operation in the future [1]. The rapid evolution of sUAS technology has made many safety, commercial, and recreational applications possible.

The management of millions of operational sUAS, in terms of keeping sUAS from colliding with terrain and with each other is expected to be accomplished outside of existing air traffic management provision applied to controlled airspace. sUAS will be restricted to low altitudes (up to 400 feet above ground level (AGL)) and operate outside of controlled airspace. sUAS operating outside of these restrictions will be subject to the same air traffic regulations applied to all aircraft in controlled airspace. sUAS operating within these restrictions will be able to take advantage of the UTM concept under development by NASA, the Federal Aviation Administration (FAA) and many others.

To accomplish management of sUAS traffic in a UTM environment, $\mathrm{C} 2$ communications must be robust and reliable, have high availability, and also be affordable and easy to implement. While not requiring the level of performance expected for operations in controlled airspace using aviation safety spectrum, C2 for UTM must nevertheless perform reliably to provide a sufficient level of system safety.

UTM C2 communications links are expected to rely heavily on existing commercial cellular networks, such as fourth generation of broadband cellular network technology (4G) and Long-Term Evolution (LTE) standard systems, which can provide an availability and cost level commensurate with the size, 
weight, power and economics of sUAS. In addition, sUAS control links operating in the industrial, scientific, and medical (ISM) radio bands can be applied to the UTM environment.

Within NASA's UTM project, the potential performance of these UTM C2 links and networks is under study. An important aspect of this study is the understanding of the potential impact of the electromagnetic interference environment on sUAS C2 link performance. In addition to simulation and analysis of $\mathrm{C} 2$ link and network performance, flight experiments are planned to characterize the RF environment at altitudes up to $400 \mathrm{ft}$ to provide real world data.

The flight experiments will use an RF channel sensing payload attached to an sUAS. This payload has been under development at NASA's Glenn Research Center. Initial flight tests will occur at NASA's Ames Research Center in the Spring of 2018.

The following sections of this paper will provide an overview of the UTM project and the types of command and control links being tested and demonstrated in the UTM project under several Technology Capability Level (TCL) testing activities, in particular TCL-3 which includes a focus on communications, navigation and surveillance (CNS) performance. This is followed by a description of the RF channel sensing payload design, test plans and initial payload and site survey test results.

\section{UTM Project}

To safely and efficiently integrate the expected numbers and types of sUAS operations at low altitudes in large-scale, NASA has undertaken the development of the UTM research platform [2, 3]. The UTM platform is used to research and develop promising technologies and data exchange protocol to support routine and widespread execution of present and future envisioned applications such as urban area package delivery and infrastructure inspection.

The UTM platform also allows for conceptual and technical research that can be transferred to the FAA in the form of airspace integration requirements for further testing. For this transfer, NASA and the FAA formed the UTM Research Transition Team (RTT) with goals to: 1) research and mature increasingly complex UTM operational scenarios and technologies; 2) demonstrate those capabilities on the
NASA UTM research platform; and 3) deliver to the FAA technology transfer packages that enable NAS service expectations for low-altitude airspace operations by providing insight and capability requirements for critical services. [4]

\section{UTM Technology Capability Level Activities}

Activities taking place within the UTM research platform have been organized into a series of Technology Capability Levels (TCL), each increasing in complexity. [5]

UTM TCL-1 concluded field testing in August 2015 and is undergoing additional testing at an FAA site. Technologies in this activity addressed operations for agriculture, firefighting and infrastructure monitoring, with a focus on geofencing, altitude "rules of the road" and scheduling of vehicle trajectories.

UTM TCL-2, completed in October 2016, leveraged TCL-1 results and focused on beyond visual line-of-sight operations in sparsely populated areas. Researchers tested technologies that allowed dynamic adjustments to availability of airspace and contingency management.

UTM TCL-3 will occur in Spring 2018, leveraging TCL-2 results and focusing on technologies that maintain safe spacing between cooperative (responsive) and non-cooperative (nonresponsive) sUAS over moderately populated areas.

UTM TCL-4, with dates to be determined, will leverage TCL-3 results and focus on UAS operations in higher-density urban areas for tasks such as news gathering and package delivery. It will also test technologies that could be used to manage large-scale contingencies.

Testing occurs at six UAS test sites located in Alaska, North Dakota, Nevada, New York, Texas and Virginia [6].

\section{TCL-3 CNS Testing}

TCL-3 activities focus on 4 areas: Communication, Navigation, and Surveillance; Sense and Avoid; Data and Information Exchange; and Concepts.

Three areas of testing under Communications, Navigation and Surveillance (CNS) cover the following: 
Test CNS1 - Maintaining control of the UA with a redundant $\mathrm{C} 2$ link. The purpose is to evaluate effectiveness of redundant $\mathrm{C} 2$ links in maintaining operational control of UA.

Test CNS2 - Remaining within Flight Geography using GNSS Navigation. The purpose is to evaluate the impact of GNSS navigation error on UA's ability to stay within the flight geography.

Test CNS3 - RF Interference Baseline Monitoring. The purpose is to Characterize RF environment UA operate in and evaluate its impact on UA's C2 link.

CNS testing in TCL-3 will occur at all test sites except Virginia.

\section{UTM TCL-3 C2 Links}

The UAS test sites where TCL-3 activities will occur will be deploying a number of different sUAS platforms using several forms of communications for C2. These include commercial LTE networks, high bandwidth line-of-site ISM Band communications, Wi-Fi (2.4 GHz and 5.8 GHz), VHF, and amateur band $(900 \mathrm{MHz})$ command and control, as well as Iridium LEO satellite communications links. For surveillance, ADS-B, and TIS-B are used. Navigation infrastructure includes GPS and radar.

Table 1 shows the LTE/4G frequency bands potentially available for UTM C2 communications via commercial cellular networks, some of which will be used for TCL-3 research and test activities.

\section{C2 Issues Under Study}

Two of the primary communications links under consideration for UTM C2 are commercial LTE/4G networks and ISM band line-of-sight links.

As commercial users on the ground and sUAS in flight will share the same LTE communications network, capacity and service prioritization will need to be analyzed. Of principal concern is the behavior of cell technology in the face of sudden high demand, which may lead to dropped links - in the case of UTM a C2 link failure could result. The possibility of prioritization of UTM communications flows to serve in flight sUAS can be examined. Other LTE-based C2 parameters of interest in determining $\mathrm{C} 2$ link include quality of service (data integrity, latency), handoff
Table 1 - LTE/4G Bands

\begin{tabular}{|l|l|l|}
\hline Band & $\begin{array}{r}\text { Base Station } \\
\text { Transmit Bands }\end{array}$ & \multicolumn{1}{|c|}{$\begin{array}{c}\text { User } \\
\text { Equipment Transmit } \\
\text { Bands }\end{array}$} \\
\hline $700 \mathrm{MHz}$ & $717-768 \mathrm{MHz}$ & $\begin{array}{l}699-716 \mathrm{MHz} \\
777-798 \mathrm{MHz}\end{array}$ \\
\hline $800 \mathrm{MHz}$ & $832-869 \mathrm{MHz}$ & $807-824 \mathrm{MHz}$ \\
\hline $850 \mathrm{MHz}$ & $852-894 \mathrm{MHz}$ & $814-849 \mathrm{MHz}$ \\
\hline $1700 \mathrm{MHz}$ & $\mathrm{N} / \mathrm{A}$ & $1710-1780 \mathrm{MHz}$ \\
\hline $1900 \mathrm{MHz}$ & $1930-1995 \mathrm{MHz}$ & $1850-1915 \mathrm{MHz}$ \\
\hline $2100 \mathrm{MHz}$ & $2110-2170 \mathrm{MHz}$ & $1920-1980 \mathrm{MHz}$ \\
\hline $2300 \mathrm{MHz}$ & $2350-2360 \mathrm{MHz}$ & $2305-2315 \mathrm{MHz}$ \\
\hline $2500 \mathrm{MHz}$ & $2496-2690 \mathrm{MHz}$ & $2496-2690 \mathrm{MHz}$ \\
\hline
\end{tabular}

between cell towers, handoff between providers, and impact of protocol variations. In addition, general system availability, coverage gaps, and in the case of hybrid terrestrial/satellite $\mathrm{C} 2$ systems, the handoff performance between terrestrial and satellite communications systems are also significant issues affecting potential C2 system performance. Finally, cybersecurity aspects that may potentially affect UTM C2 systems must be accounted for.

Both ISM band links and LTE network-based links can have potential interference problems. When operating at altitude, sUAS will have a much longer radio line-of-sight compared to a user on the ground. Many more LTE/4G towers will be visible and many more ISM band transmitters will be visible. Therefore, characterization of the RF environment at altitudes up to 400 feet AGL is needed to understand the RF interference potential.

\section{RF Channel Sensing Payload}

A key aspect of UTM C2 communications involves the application of commercial networks and the expected performance. Since several commercial networks can provide $\mathrm{C} 2$ services, and these networks operate in a competitive environment, the available performance information is limited. Even less information is known regarding the performance of these commercial networks at altitudes up to 400 feet, that is, in the UTM environment. Therefore, NASA is interested in better understanding these performance issues, as described in the previous section.

The UTM Project's TCL-3 testing activities include characterization of the RF environment under Test CNS3. In addition to the test sites where CNS tests 
will be conducted, NASA is also studying the $\mathrm{C} 2$ link performance, including the development and deployment of an RF channel sensing payload.

The RF channel sensing payload, developed by the NASA Glenn Research Center, is designed to allow the measurement of signals in the LTE/4G and ISM bands intended to support future UTM C2 links (Table 1). The payload will characterize the RF environment at altitudes up to 400 feet above ground level (AGL). Future upgrades of the payload will have added capabilities to analyze communications performance parameters, allowing link performance to be correlated with the RF environment.

The RF channel sensing payload is implemented using a software defined radio (SDR), operated with GNU Radio Companion shareware software [7]. This software enables complicated transceiver designs to achieve the needed communication capability. For this task the Ettus ${ }^{\mathrm{TM}}$ Models E310/E312 SDR was selected [8]. The E310/312 models were chosen because they have a larger Xilinx field programmable gate array (FPGA) allowing for more customized FPGA block processing. The primary difference between the E310 and E312 is that the E312 includes an integrated battery, while the E310 requires an external battery. The E312 has been tested to run 2 to 3 hours, and without the external battery allows for a lighter and more compact payload, which can be required depending on the sUAS platform available to carry the payload.

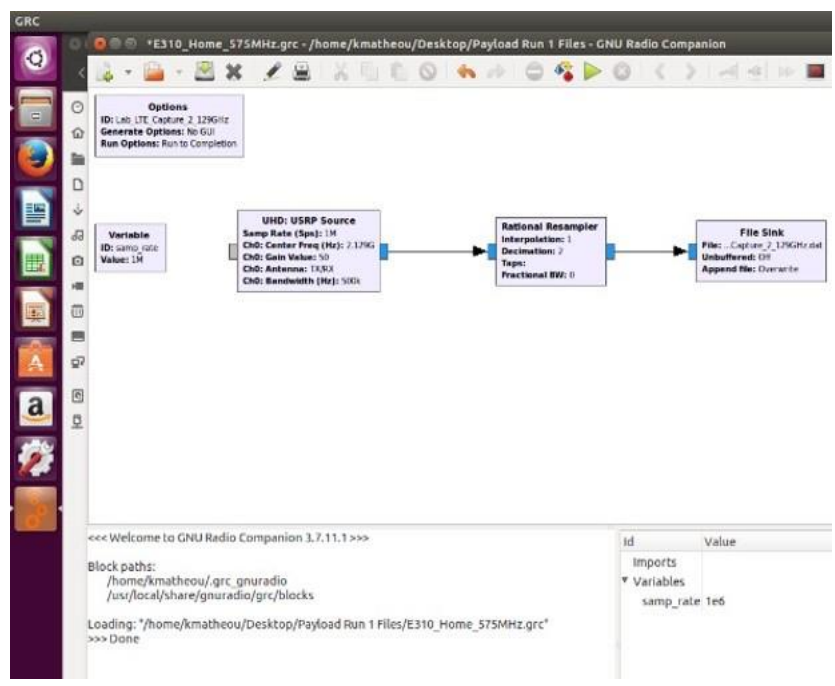

Figure 1 - GNU Flowgraph

\section{Payload Design}

The current SDR design has been programmed using the GNU Radio Companion freeware software. The GNU software runs on Linux OS based machines. The GNU software uses function box type modules to create flowgraphs. Once a flowgraph is correctly created, the GNU software creates python code, which is then transferred to the E310/312 via Ethernet communication. Figure 1 is an example of such a flowgraph design.

Due to the internal limitations of writing complex samples to the E310/312 removable micro SD card, by experimentation it was found the sample rate writing to micro SD card limit for the E310/312 is approximately $500 \mathrm{kHz}$ complex bandwidth (BW). The micro SD card supplied with the E310/312 is an 8 GB card. After the Linux embedded system and all GRC related software overhead are added, about 2 GB of space remains. From experimentation, it was found that at the complex sample capturing rate of $500 \mathrm{kHz}$ (I and Q samples are 12-bit resolution from the ADC transferred to 16-bit when written to the hard drive), only 10 to 12 minutes of recording complex data will fit on the remaining space of the micro SD card.

\section{Payload Operation}

The E310/312 payload and connection diagram are shown in Figure 2. Before takeoff, the SDR unit is connected to a Linux laptop computer via an $1 \mathrm{G}$ Ethernet cable. Assuming that the python code is already saved on the microSDcard, the Linux laptop will initiate the code to start running. Currently, there is no WiFi type communication to the $\mathrm{E} 310 / 312$, so by the time the Ethernet cable is unplugged from the payload and everyone is in a safe distance for takeoff, a hard coded 2 minute delay before recording sample data is programmed.

Once the sUAS and payload are in the air, measurements will be made at several altitudes such as: $50 \mathrm{ft}$., $150 \mathrm{ft}$. and $400 \mathrm{ft}$ AGL. Most LTE standalone towers range between 100-140 ft. AGL. Thus, with these initial starting points 3 levels should provide sufficient information about relative LTE spectrum and signal strengths at each altitude.

After 12 minutes of flight, the sUAS will land. The sample data is recovered by ejecting the micro SD card, and then post-processed using a Matlab code 


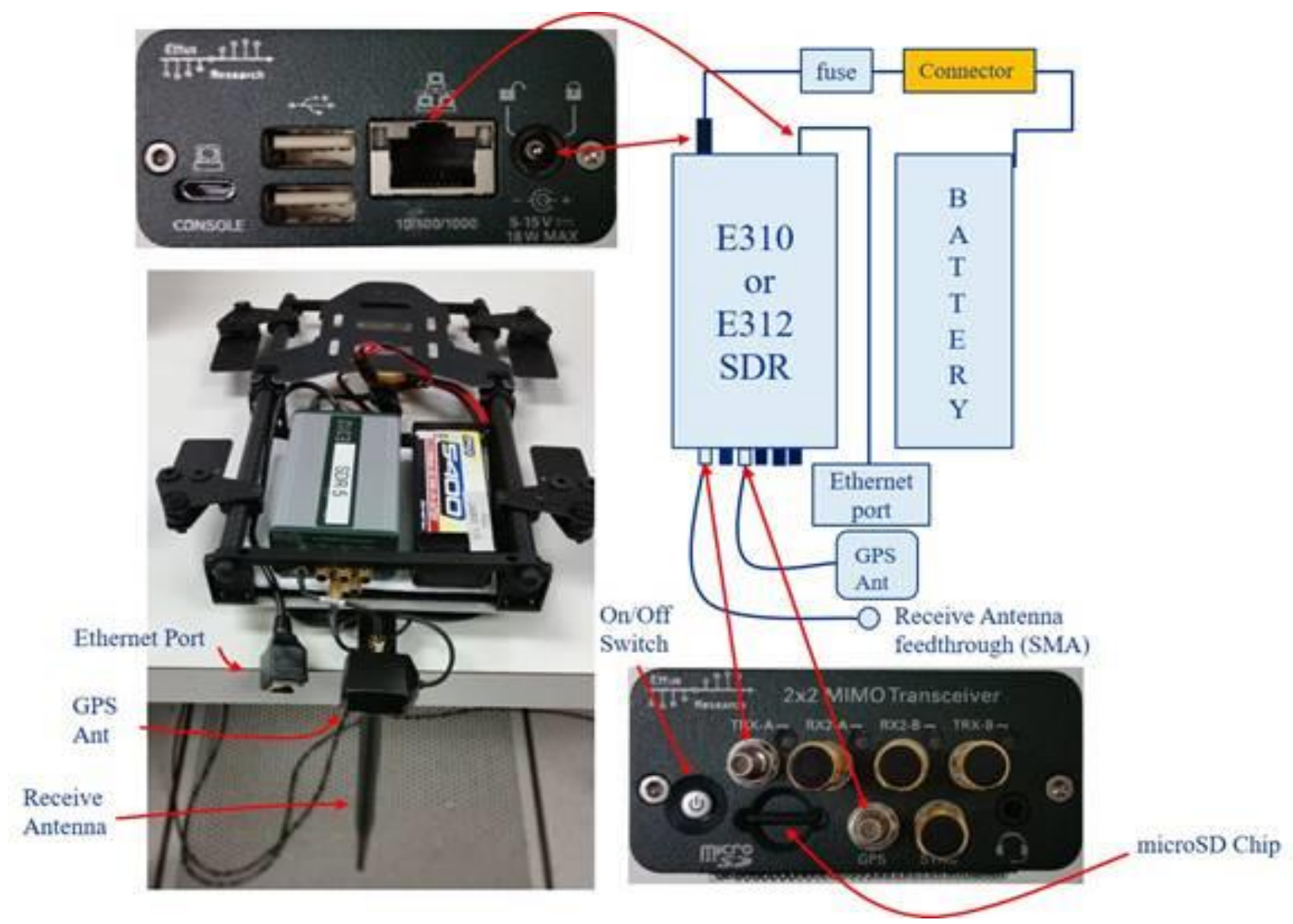

Figure 2 - the RF Channel Sensing Payload (left, center) and Connection Diagram.

where the complex samples are read and plotted as shown in the following sections.'

\section{Payload Performance}

Prior to the planned initial flight testing in early Spring 2018, RF spectrum measurements have been captured in the laboratory at NASA Glenn to test the process of utilizing the SDR and capture free space spectrum data.

Figure 3 is a plot of the LTE/WiFi spectrum covering $2.1 \mathrm{GHz}$ to $2.15 \mathrm{GHz}$ recorded in the lab by a spectrum analyzer. The resolution $\mathrm{BW}$ is $1 \mathrm{kHz}$, and the overall $\mathrm{BW}$ is $50 \mathrm{MHz}$. To make a comparison with an RF channel sensing payload's $500 \mathrm{kHz}$ measurement capability, a reduced $500 \mathrm{kHz}$ portion of the spectrum analyzer plot is shown in Figure 4. We chose the $2.129 \mathrm{MHz}$ center frequency where we see the edge of the LTE spectrum for contrast. The same spectrum was then captured using the RF channel sensing payload using a measurement time of three minutes, capturing 7.2 million complex samples. A Matlab program was used to create discrete Fourier transform (DFT) resulting in the plot shown in Figure 5.

It is important to note that the E310/312 produces the sampled data as complex baseband. The SDR is a direct conversion SDR. Thus, the IF frequency is removed and the spectrum should always be shown in complex bandwidth format to make sure both sides of the spectrum are measured, as in Figure 5. Comparison with the spectrum analyzer measurement in Figure 4 shows a similar spectrum. Note that the dynamic range between the noise floor and the LTE/WiFi signal is equal at $10 \mathrm{~dB}$ in dynamic range. Since the E310/312 is writing at its maximum sample rate to the micro SD card, there appear a few overrun signals producing some spikes that can be ignored. The roll off observed in Figure 5 on the left and right sides of the plot results from the E310/312 IF down converting and filtering. This is a limitation of the current implementation using this SDR. Further development, taking advantage of the E310/312 complex processing capability will add a DFT firmware based block to the 


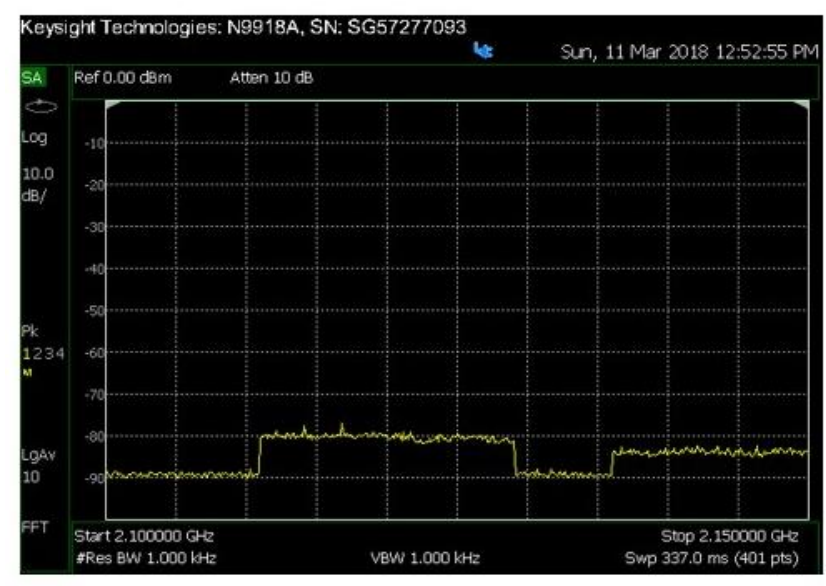

Figure 3 - Spectrum Analyzer Plot of LTE/ WiFi Spectrum, 2.125 GHz, $50 \mathrm{MHz}$ Span

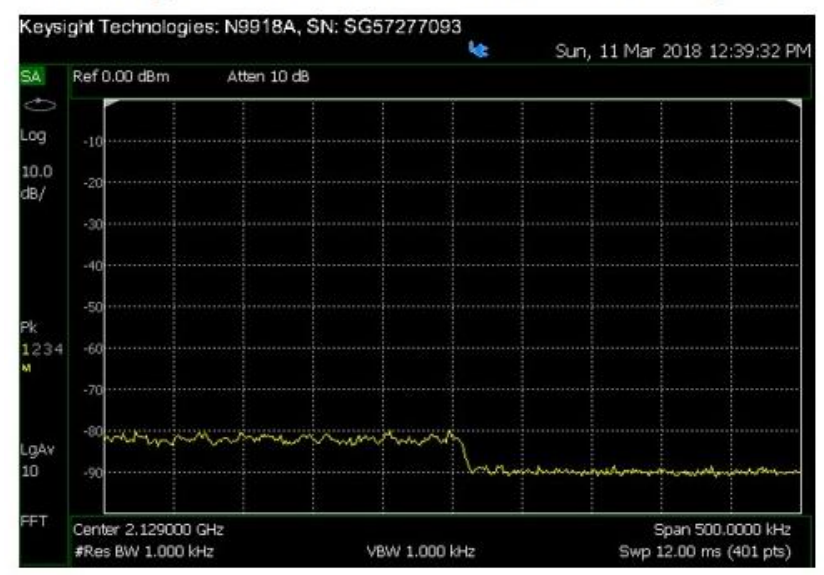

Figure 4 - Spectrum Analyzer Plot of LTE/ WiFi Spectrum, 2.129 GHz, 500 kHz Span

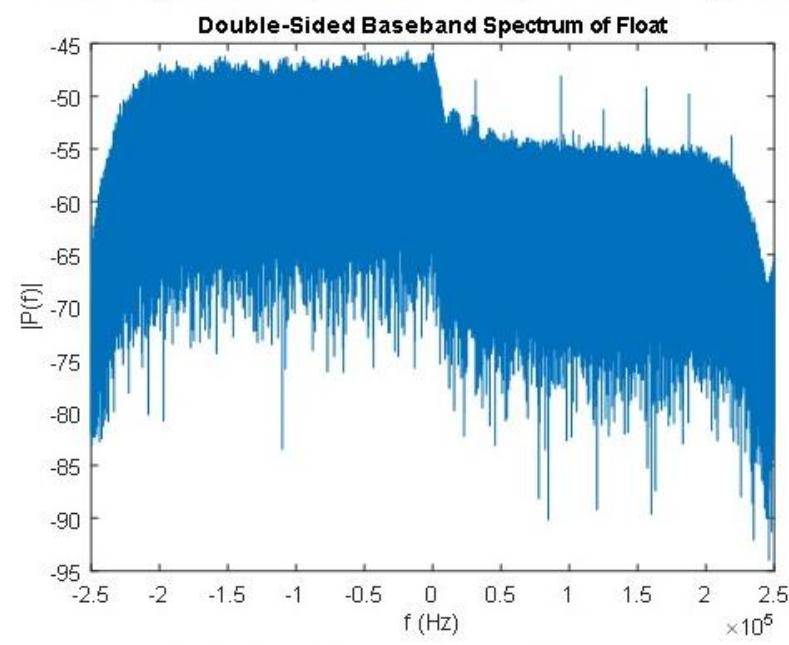

Figure 5 - RF Channel Sensing Payload Measurement of LTE/WiFi Spectrum, 2.129 GHz, 500 kHz Span
FPGA image enabling larger bandwidths to be captured.

\section{Payload - sUAS Integration}

The vehicle intended to carry the RF channel sensor payload is a Dà-Jiāng Innovations Science and Technology Co., Ltd (DJI) model S1000, operated by NASA Ames Research Center. This vehicle has a 40A electronic speed controller (ESC) built into each arm in an octocopter configuration. The 4114 pro motors, high performance 1552 folding propellers, and V-type mixer design combine to give each arm of the S1000 a maximum thrust of $2.5 \mathrm{Kg}$. The vehicle's frame arms and landing gear are made from carbon fiber. Figure 6 is a picture of the vehicle [9].

The RF channel sensing payload, consisting of the E310/312, external battery, receive antenna, and connections is shown in Figure 2, in the left center photograph. The components are mounted to an aluminum plate connected to a mounting kit compatible with the S1000. As shown in Figure 2, the receive antenna points down, with the payload mounted at the bottom of the S1000. Figure 7 shows three views of the payload mounted to the S1000.

A series of electromagnetic interference (EMI) tests was conducted on the DJI S1000 in the NASA Ames RF Test Lab during January 30-February 1, 2018. The purpose was to determine if any EMI originating from the $\mathrm{S} 1000$ while in operation is present within the frequency bands to be tested by the RF channel sensing payload. As reported in [9], no EMI signals were observed in those bands.

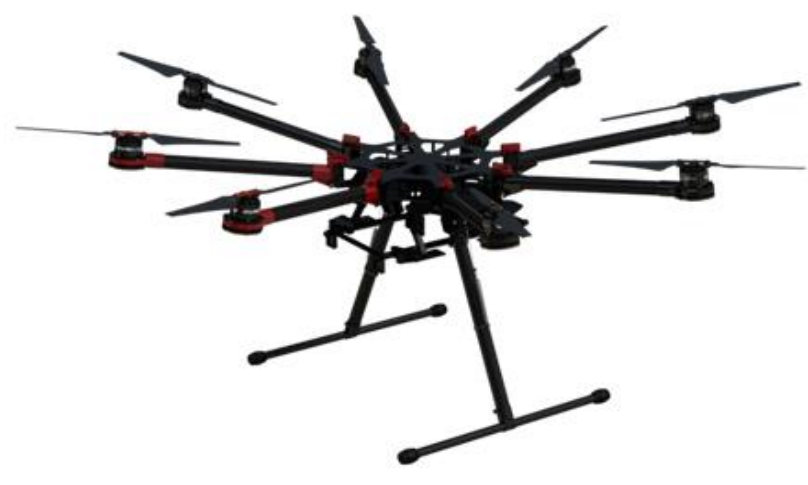

Figure 6 - Picture of S1000 


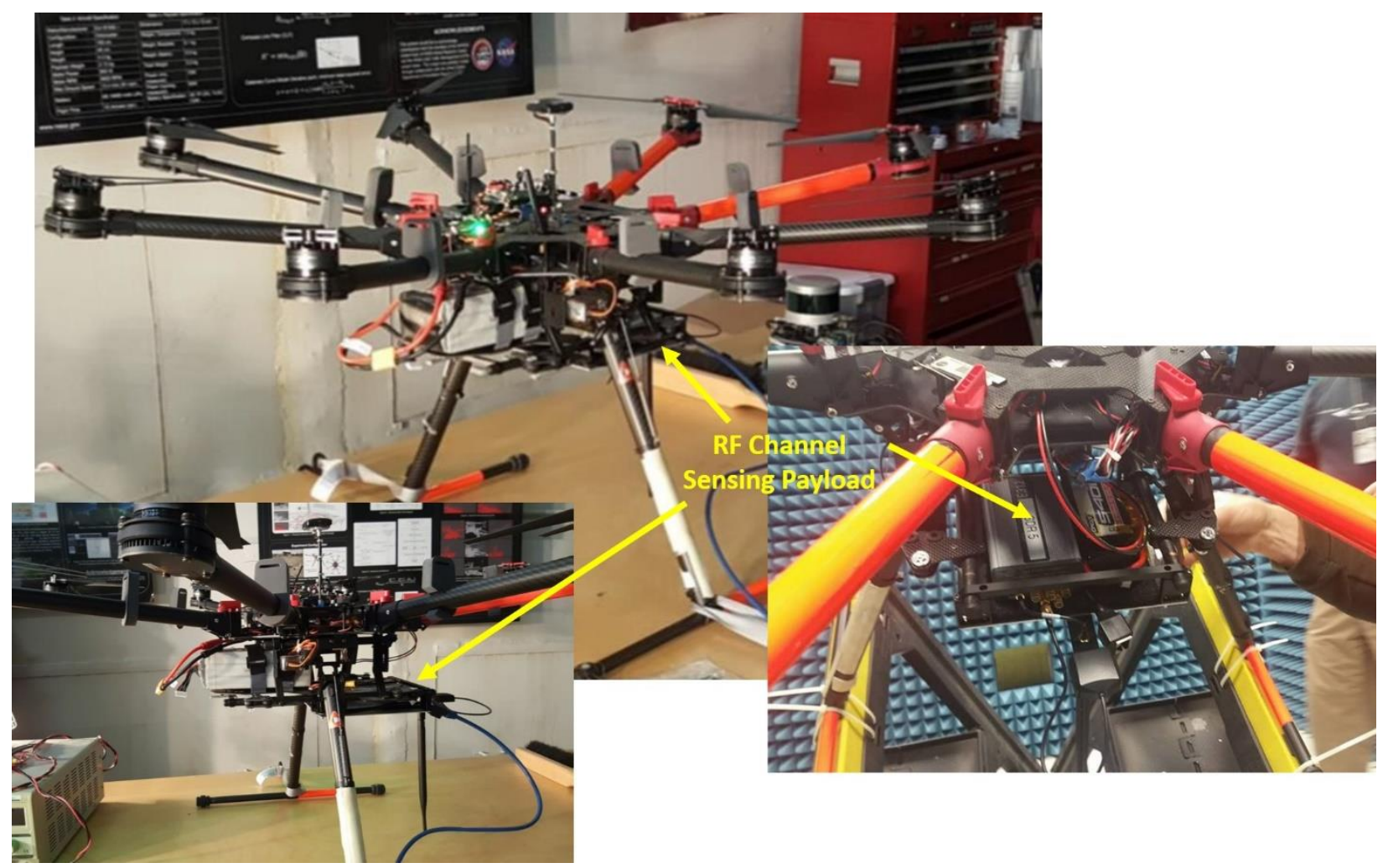

Figure 7 - Three Views of the RF Channel Sensing Payload Integration with the DJI S1000

\section{Flight Testing}

\section{Flight Test Approach}

Initial flight testing will take place at NASA Ames Research Center/Moffett Field, California during Spring 2018. The principal objective of this trial is to evaluate three-dimensional spectrum coverage and signal strength provided by commercial wireless communications service providers in the vicinity of the test locations. Specifically, this test section will evaluate the performance of LTE and $4 \mathrm{G}$ base station transmissions at designated altitudes and geolocations.

The test plan design currently under development considers the known location of commercial cellular service provider tower. A preliminary survey of the test area revealed the presence of AT\&T, Verizon, Sprint and T-Mobile antenna systems located near testing locations.

RF testing approach will consider samples at several altitudes between 0 and $400 \mathrm{ft}$. AGL utilizing an omnidirectional antenna. The test grid represented in Figure 8 shows the altitudes versus possible test campaigns. The RF channel sensing payload will be flown aboard the DJI S1000 flight vehicle to the several altitudes at three or more physical locations. The S1000 will be commanded to hover at a given altitude while the payload takes RF spectrum data in the bands of interest. Each flight will capture data from a specific frequency band.

Ground measurements of the RF environment will guide selection of the airborne frequencies of interest to optimize flight battery limitations. The trials will focus on measuring signal strength transmitted by

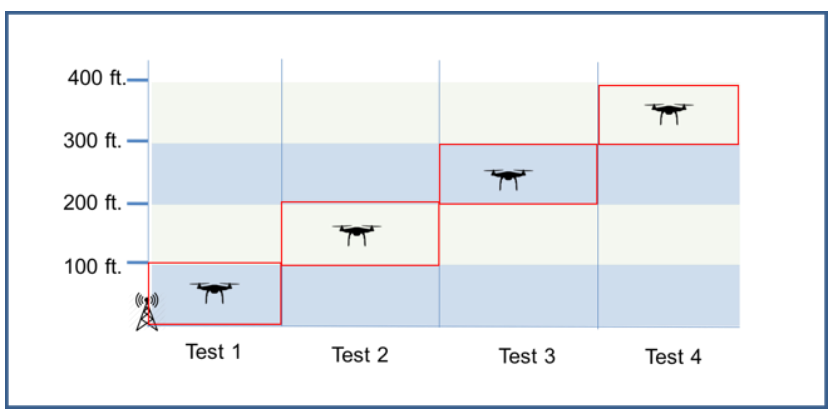

Figure 8 - Altitude vs. Location Test Grid 


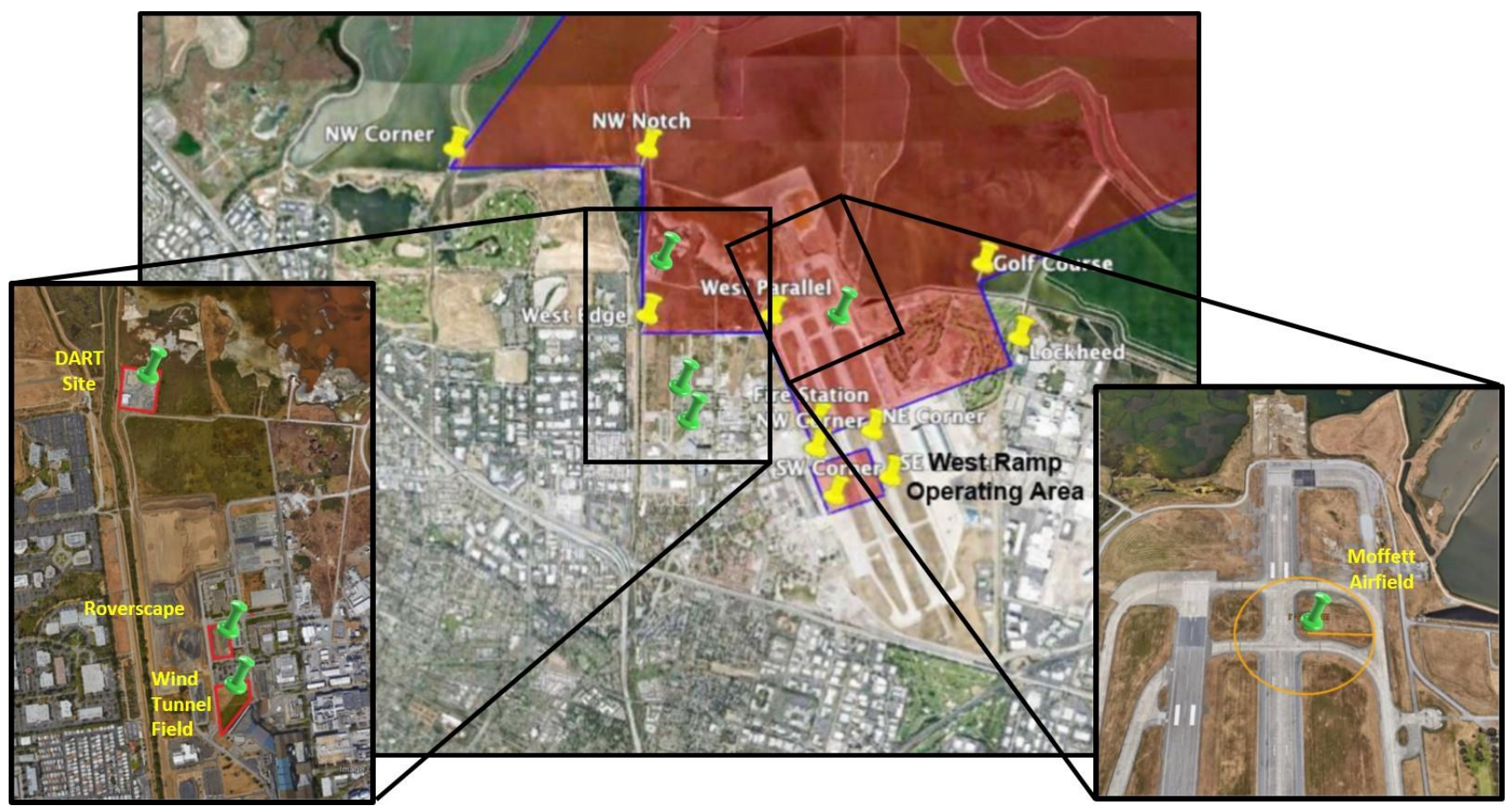

Figure 9 - Possible Flight Test Locations at NASA Ames Research Center/Moffett Field. Image (CGoogleEarth [10]

base station equipment. User equipment (UE) transmissions will not be measured during these tests.

Possible flight test sites are shown in Figure 9 [10]. The Moffett Airfield location allows untethered flight testing, while the DART Site, Roverscape and Wind Tunnel Field may require tethered operations.

The preferred test site would enable the payload to capture all 4 main service providers of LTE signals. However, more than one site will likely need to be tested. At each site, the objective of the flight test is to capture enough spectrum data at 2 or 3 different altitudes at each service provider's LTE frequency range, the maximum altitude being 400 feet AGL. The average AGL LTE tower height is 120 feet. Flights are expected to average about 12 minutes based on the S1000 flight time and payload battery life.

For initial testing, a smaller bandwidth will be captured and each payload flight trip will be focused on one LTE carrier's 'sweet spot' range. The sweet spot range is determined by the main LTE channel range is and the respective $\mathrm{BW}$. Once they are determined, the center frequency range will be chosen where most LTE overhead communication occurs. Once the flight is completed, a SDR text file will be created, input to a Matlab program that will parse the complex sample data and analyze the spectrum per altitude per a specific BW spectrum.

\section{Flight Test Site Survey}

Three possible flight test sites at NASA Ames Research Center/Moffett Field were surveyed during January 30-February 1 2018: Moffett Airfield, the Wind Tunnel Field and the DART Site (see Figure 8). The site surveys considered physical suitability of the location for the RF channel sensing payload flight tests, and also included spectrum sensing surveys using a handheld spectrum analyzer to determine the presence of measurable signals. Using CellReception [11], cellular service provider tower locations and heights in the nearby area were determined. This information will assist in the analysis of the captured spectrum complex samples. Of course, the capture is at ground level, but it allows the team to understand what is existing at ground level and will compare dynamic ranges of signals in several capture altitudes up to $400 \mathrm{ft}$ AGL. Figure 10 shows an example of a spectrum analyzer measurement at the DART site. Signals in the 700-850 MHz LTE band are observed in the measurement, indicating a good potential site for the initial flight testing scheduled for Spring, 2018. 


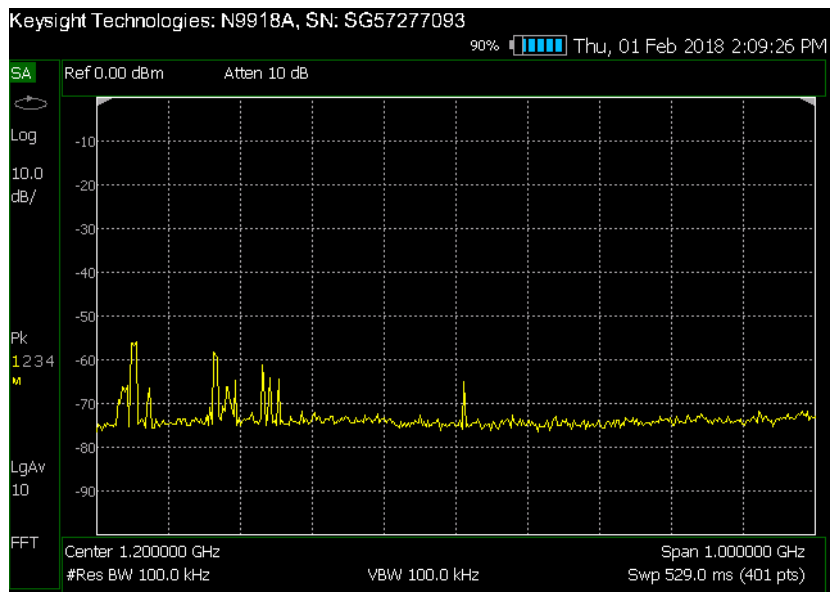

Figure 10 - Spectrum Analyzer Measurement of Ambient Signals at the DART Test Site.

\section{Summary}

The NASA UTM Project has been undertaken to enable the safe and efficient integration of small UAS operations in large-scale at low altitudes. Test and research activities are being conducted at six UAS test sites in the United States.

A key element under study is the performance of UTM C2 links. A number of performance parameters of interest are being studied, in particular, the performance of $\mathrm{C} 2$ links based on commercial LTE/4G networks at altitudes up to $400 \mathrm{ft}$. AGL. UTM research activities are organized around technology capability levels (TCL), with TCL-3 occurring in 2018. CNS tests will occur in TCL-3 including characterization of the RF environment.

NASA is augmenting the TCL-3 activities with the development and deployment of an RF channel sensing payload. The payload, based on SDR technology, will fly on a DJI S1000, with first flight tests occurring in Spring, 2018.

Laboratory testing has confirmed the capability of the RF channel sensing payload to capture RF spectrum in several frequency bands of interest, in 500 $\mathrm{kHz}$ segments. The payload will be further developed to increase the measurement bandwidth and add capabilities to analyze communications performance parameters, allowing $\mathrm{C} 2$ link performance to be correlated with the RF environment.

\section{References}

[1] FAA Aerospace Forecast Fiscal Years 2017-2037, Publication, FAA, Washington, D.C., 2016.

[2] Kopardekar, P., Rios, J., Prevot, T., Johnson, M., Jung, J., and Robinson, J. E., "Unmanned Aircraft System Traffic Management Concept of Operations," 16th AIAA Aviation Technology, Integration, and Operations Conference, Washington, D.C., 13-17 June 2016.

[3] Kopardekar, P., "Unmanned Aerial System Traffic Management (UTM): Enabling Lowaltitude Airspace and UAS Operations," Tech. Rep. NASA TM-2014-21829, 2014.

[4] UAS Traffic Management (UTM) Research Transition Team (RTT) Plan, Publication, FAA, Washington, D.C., 2017.

[5] Unmanned Aircraft System (UAS) Traffic Management (UTM)

https://utm.arc.nasa.gov/index.shtml]

[6] Rios, J., Mulfinger, D., Homola, J., Venkatesan, P., "NASA UAS Traffic Management National Campaign", Digital Avionics Systems Conference 2017, September 17-21, 2017, St. Petersburg, FL.

[7] GNU Radio Companion

https://wiki.gnuradio.org/index.php/GNURadioComp anion

[8] Ettus Research ${ }^{\mathrm{TM}}$ https://www.ettus.com/ content/files/USRP_E310_Datasheet.pdf

[9] Jung, J., Ippolito, C., Rogers, C., Kerczewski, R., Downey, A., Matheou, K., "Small Unmanned Aircraft Electromagnetic Interference (EMI) Initial Assessment", 2018 ICNS Conference, April 2018, Herndon, VA.

[10] Map data @2016 Google. Google, Inc., 1600 Amphitheatre Parkway, Mountain View, CA 94043, http://www.google.com/earth/

[11] CellReception, ttp://www.cellreception.com

2018 Integrated Communications Navigation and Surveillance (ICNS) Conference

April 10-12, 2018 